\title{
Constant Positional Control by GA of an Airship-type Robot Propelled by Pectoral Fin Movement
}

\author{
Tadashi Goto* Non-member \\ Masafumi Uchida* Member
}

We report herein the constant positional control by GA of an airship-type robot propelled by pectoral fin movement.

Keywords: pectoral fin, entertainment robot, fish robot, flying robot, genetic algorithm

\section{Introduction}

We have developed an airship-type robot that hovers in the air and has a propulsions mechanism that resembles a fish swimming through the air. We considered pectoral fins as the propulsion mechanism for an airshiptype robot. The pectoral fins are chiefly used in operation and in stabilization of the body. Using pectoral fin movement as the propulsion mechanism of the airshiptype robot, we have developed a robust constant position control system. We use a genetic algorithm (GA) for position control and attempted an autonomous search to determine the best pectoral fin movement pattern. However, when the GA is applied to an actual robot, a problem exists in that the time required to search for an optimum answer is significant. Therefore, we attempted to improve the settling performance of the GA by performing the actual operation and the simulation in parallel.

\section{Specifications of Airship Type Robot}

Figure 1 shows the structure of the robot. The robot is has a buoyancy of approximately $200 \mathrm{~g}$, which is provided by a balloon filled with helium. The pectoral fin mechanism, which consists of two servo motors (GWS PICO BB) and two fins constructed of sheets of polystyrene foam, a sensor unit containing three ultrasonic sensors (BestTechnology BTE054) and one yaw sensor (MicroStone MGQ1-01B), and an H8 microcomputer (AKI-H8/3048F) are installed in the frame that supports the balloon ${ }^{(1)}$. Figure 2 shows the control system. The $x, y$, and $z$ axes are defined as shown in Fig.2. The $x, y$, and $z$ coordinates of the robot are measured using three ultrasonic sensors, and the yaw angle $\theta$ of the robot is measured using a yaw sensor. Data is sent and received by the $\mathrm{H} 8$ microcomputer through an $\mathrm{RS} 232 \mathrm{C}$ cable connected to a PC. The power supply is an external supply.

\section{Constant Positional Control by GA}

Figure 3 shows the control flow chart. The GA searches for the best pectoral fin movement pattern by which to move the robot to the goal position. The

\footnotetext{
* The University of Electro-Communications

1-5-1, Chofu-ga-oka, Chofu 182-8585
}

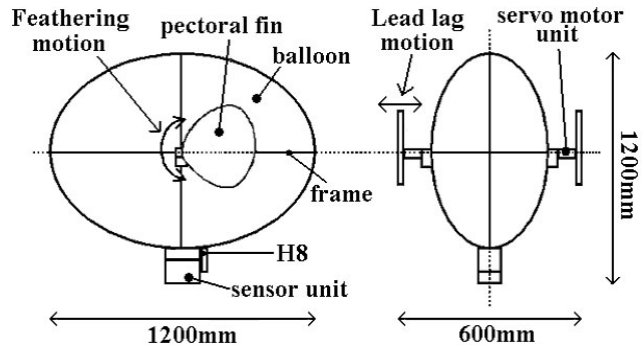

Fig. 1. Structure of the robot

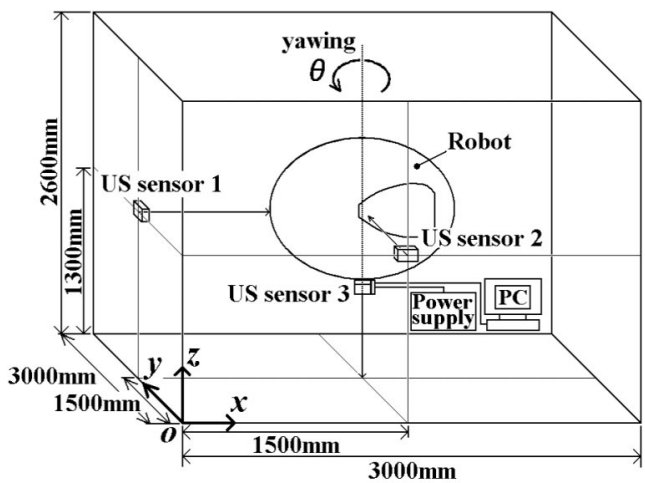

Fig. 2. Control system

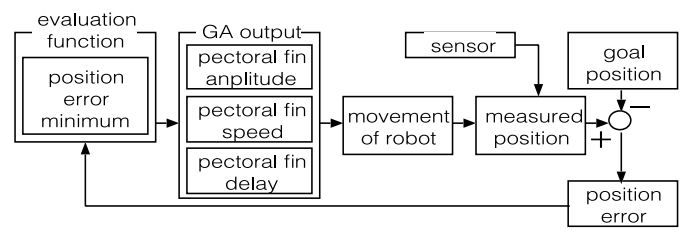

Fig. 3. Control flow chart

initial position is assumed to be $P_{0}=\left(x_{0}, y_{0}, z_{0}, \theta_{0}\right)$, and the goal position is assumed to be $P_{g}=\left(x_{g}, y_{g}, z_{g}, \theta_{g}\right)$. When the robot is made to move from position $P_{0}$ for time $t$ using a certain movement pattern, the position of robot is assumed to be $P_{k}=\left(x_{k}, y_{k}, z_{k}, \theta_{k}\right)$. The evaluation function $F$ is used to evaluate the movement pattern and determine the error margins of $P_{g}$ and $P_{k}$. The evaluation function $F$ is given by the following equation:

$$
F=\frac{a}{1+g_{x}\left(x_{g}-x_{k}\right)+g_{y}\left(y_{g}-y_{k}\right)+g_{z}\left(z_{g}-z_{k}\right)+g_{\theta}\left(\theta_{g}-\theta_{k}\right)}
$$


where $a$ is an arbitrary constant and $g_{x}, g_{y}, g_{z}$ and $g_{\theta}$ are the weight functions of each parameter. The individual having the highest evaluation in the final generation is considered to be the optimal solution. The best movement pattern is decided by reading the genetic information of this individual. To improve the settling performance of the GA, we used a compound GA that combines actual operation and the simulation. The GA in actual operation is assumed to be $G A_{O}$, and the evaluation function of $G A_{O}$ is assumed to be $F_{O}$. The GA in the simulation is assumed to be $G A_{S}$, and the evaluation function of $G A_{S}$ is assumed to be $F_{S}$. Moreover, the GA that brings $F_{S}$ close to $F_{O}$ is assumed to be $G A_{F}$, and the evaluation function $F_{F}$ is given by the following equation:

$$
F_{F}=\frac{b}{1+\left|F_{O}-F_{S}\right|} \cdots \cdots \cdots \cdots \cdots \cdots \cdots
$$

where $b$ is an arbitrary constant. The result of $G A_{S}$ can be reflected directly in $G A_{O}$ by bringing $F_{S}$ close to $F_{O}$. Because the time required to actually make the robot function can be omitted, the optimization time can be greatly reduced.

\section{Constant Position Control Experiment}

In the following, we consider the $z$ direction. The position is adjusted to a relative position. Here, $z_{0}=0 \mathrm{~cm}$, $z_{g}=100 \mathrm{~cm}, t=5 \mathrm{~s}$, and $F_{O}$ is given by the following equation:

$$
F_{O}=\frac{1000}{1+\left|z_{g}-z_{k}\right|} \cdots \cdots \cdots \cdots \cdots \cdots \cdots \cdots
$$

In the present study, we examined the case of using only $G A_{O}$ and the case of using only the compound GA, respectively. The case of $G A_{O}$ is denoted as case A. In case $\mathrm{A}$, the robot is actually made to function by $G A_{O}$ until the tenth generation. The cases in which only the compound GA is used are denoted as cases B, C and D. The robot is actually made to function by $G A_{O}$ until the 1st generation in case $\mathrm{B}$, until the 5 th generation in case $\mathrm{C}$, and until the 9 th generation in case $\mathrm{D}$. The optimization of 200th generation is performed by $G A_{S}$ using the $F_{S}$ obtained by $G A_{F}$. Afterward, the optimal solution obtained by $G A_{S}$ is reflected in $G A_{O}$. In each condition, we measured the change in the $z_{k}-z_{g}$ distance, and the experiment was conducted five times. Next, for the cases of $z_{g}=0 \mathrm{~cm}, 25 \mathrm{~cm}, 50 \mathrm{~cm}, 75$ $\mathrm{cm}$, and $100 \mathrm{~cm}$, the simulation of the 200th generation in $G A_{S}$ was performed using $F_{S}$ obtained by D. The result is reflected in $G A_{O}$ by the compound GA, and we measured the $z_{k}-z_{g}$ distance. The experiment was conducted five times for each $z_{g}$.

\section{Experimental Results}

Figure 4 shows the change in the $z_{k}-z_{g}$ distance for each case. The marker is the average of five measurements, and the error bar is the standard deviation $\sigma$. The minimum distance was $40 \mathrm{~cm}$ in the tenth generation in $\mathrm{D}$, the optimization of which required $30 \mathrm{~min}$. Figure 5 shows the distance of the optimal solution for $G A_{S}$ and the distance when the optimal solution is reflected in actual operation using the compound GA. The

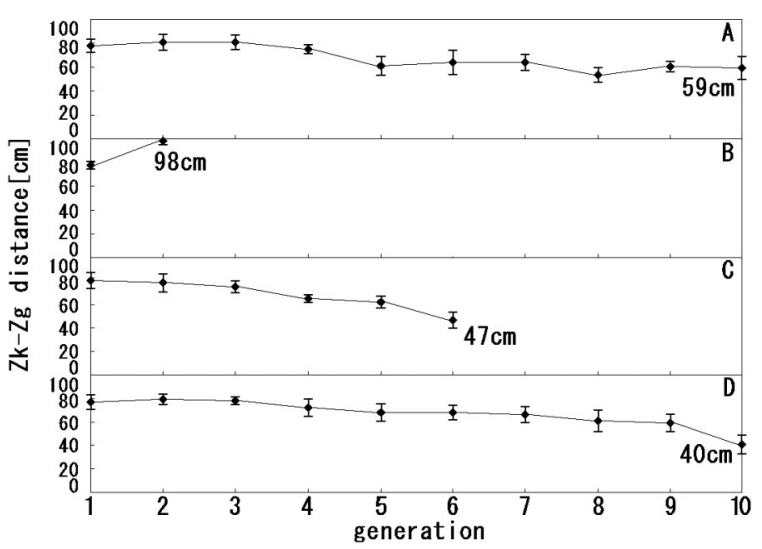

Fig. 4. Change in the distance to the goal

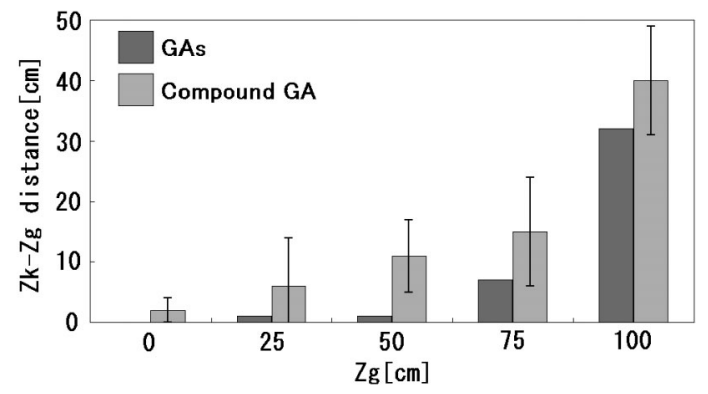

Fig. 5. Distance comparison

values are the average of five measurements, and the error bar is the standard deviation $\sigma$. The optimization for each $z_{g}$ required $5 \mathrm{sec}$. In Fig. 4 , compared to case $\mathrm{A}$, case $\mathrm{C}$ approaches $z_{g}$ in fewer generations, and the number of generations is same in cases A and D. However, among all cases, case D most closely approaches $z_{g}$. Therefore, a better pectoral fin movement pattern can be achieved in a short time by using the compound GA. In addition, the settling performance of the GA can be improved. In Fig.5, the result of the simulation by $G A_{S}$ and the result of actual operation by the compound GA are in approximate agreement. Therefore, by using the compound GA and studying $F_{S}$ beforehand, the pectoral fin movement pattern can be optimized in only 5 sec for an arbitrary height.

\section{Conclusions}

In the present report, we developed an airship-type robot propelled by pectoral fin movement, and a constant position control system by GA. Moreover, in a constant position control experiment using the compound GA, the optimization of the 200th generation, which requires eight hours using $G A_{O}$, was able to be achieved in only $5 \mathrm{sec}$. In addition, the settling performance of the GA was able to be improved. Areas for future research include power supply installation, wireless communication, and three-dimensional position control.

(Manuscript received July 11, 2005)

\section{References}

(1) N. Kato: "Control Performance in Horizontal Plane of Fish Robot in Mechanical Pectoral Fins", IEEE J. Oceanic Engineering, Vol.25, No.1, pp.121-129 (2000) 